\title{
EDITORIAL
}

\section{Health Effects of Climate Changes in Bangladesh}

(Birdem Med J 2012; 2(2): 75-76 )

The Intergovernmental Panel on Climate Change (IPCC) states clearly that climate change is contributing to the global burden of disease and premature deaths by changing weather patterns (temperature, precipitation, sea-level rise and more frequent extreme events) and indirectly through changes in the quality of water, air and food, and changes in ecosystems, agriculture, industry, human settlements and the economy.

The relationship between climate change and human health is multidimensional. IPCC 2007 shows that climate change has:• altered the distribution of some infectious disease vectors; $\bullet$ altered the seasonal distribution of some allergenic pollen species; and increased heat wave-related deaths. In 2000, climate change was estimated to have caused the loss of over160 000 lives The following health outcomes were included: • episodes of diarrhoeal disease; $\bullet$ cases of Plasmodium falciparum malaria, fatal accidental injuries caused by coastal floods and in floods/land slides; and• nonavailability of the recommended daily calorie intake( malnutrition)..

Those at greater risk include, in all countries, the urban poor, the elderly and children, traditional societies, subsistence farmers and coastal populations. Climate change is projected to increase the burden of diarrhoeal diseases in low income regions by approximately $2 \%$ to $5 \%$ in 2020.. The IPCC states that future trends in health are relevant to climate change and infectious diseases could become more prominent if public health systems are no efficient or if new pathogens arise that are resistant to our current methods of disease control, leading to falling life expectancies Bangladesh is vulnerable to outbreaks of infectious, waterborne and other types of diseases (World Bank, 2000). Records show that the incidence of malaria increased from 1556 cases in 1971 to 15375 in 1981, and from 30282 cases in 1991 to 42012 in 2004 (WHO, 2006). Other diseases such as diarrhoea and dysentery, etc. are also on the rise especially during the summer months. .These problems are thought to be closely inter linked with water supply, sanitation and food production. Climate change has already been linked to land degradation, fresh water decline, biodiversity loss and ecosystem decline, and stratospheric ozone depletion.

A changing climate is likely to affect all of these conditions• Increasing frequency of heat waves:. This has implications for Bangladesh since the elderly and children suffer the mostfrom increased temperatures. Eventhough no formal study on increase of heat waves in Bangladesh has been undertaken, we are already observing yearly trends in rise in temperatures. The health impacts associated with heat waves are heatstroke, dehydration and aggravation of cardiovascular diseases in elderly people Variable precipitation patterns: Changes in precipitation patterns are likely to compromise the supply of fresh water, thus increasing the risk of waterborne diseases. They are also associated with floods and water logging that increase the incidence of diarrhoea, cholera and skin and eye diseases. $\bullet$ Malnutrition will further increase the vulnerability of those affected to infectious and water- and vector-borne diseases. Vector-borne diseases: Changes in climate are likely to lengthen the transmission seasons of important vector-borne diseases, and alter their geographic range. Already, dengue is a regular disease in the major cities of Dhaka and Chittagong. $\bullet$ Rising sea levels: These increase the risk of coastal flooding, and may necessitate population displacement, and cause many other health-related problems such as cholera, diarrhoea, malnutrition and skin diseases, etc. More than half of the world's population now lives within $60 \mathrm{~km}$ of the sea like he Ganges-Brahmaputra delta in Bangladesh,. Incidence of major climate-sensitive

diseases occurring during the last few decades in Bangladesh Diarrhoea: 48302636 during (19882005) :annual case 2842 273.Skin diseases 23697833 
during (1988-19962) :annual cases 623 092.Malaria 1 018671 during (1974-2004) annual cases 33956 Mental disorders : 201881 during(1988-1996) annual cases 22431 Dengue : 19830 during : (1999-2005) annual case 3305 (Source: Data modified from WHO, 2006; Director- General, Health (Bangladesh); 1996, 1997; MoEF, 2005)

Ensurs priority to ensure universal accessibility to and equity in health care, with particular attention to the rural population. Efforts are being made to develop a package of essential services based on the priority needs of clients, to be delivered from a static service point, rather than through door-to-door visits Prevention and control of locally endemic diseases. Dengue: We have a national guideline. available to all practitioner. since 2004. The government introduced a National Guideline for Treatment of Malaria in1994, which was revised in 2004. Statistics from 2001 to 2005 show a marked increase in the proportion of Plasmodium falciparum cases every year. WHO declared that malaria could not be eradicated and subsequently a new strategy for malaria control was launched. The new strategy is being implemented gradually. It emphasizes disease control aspects and endorses the four technical elements (early diagnosis, prompt treatment, recognition of treatment failures and management of severe and complicated cases in hospitals) and preparedness for control of malaria outbreaks/epidemics and introduction of insecticideimpregnated bednets: Kala-azar. At least 20 million people in more than 27 districts are at risk. The major constraint is similar to that faced in the control of malaria. Eighteen million people in 12 districts are considered to be at risk of filariasis. A revised strategy for the elimination of filariasis is being pilot-tested in one district. This strategy involves administering a single dose of ivermectin with albendazole yearly for a period of three years to the total population in the district.

Though diarrhoeal diseases continue to be responsible for significant morbidity and mortality, the availability of oral rehydration solution (ORS) has increased through the formation of ORS depots in the community. Constraints include in appropriate use of anthelmintics and anti-Diarrhoeals, especially in the private sector and underutilization of health facilities.

\section{Renal Denervation Therapy (RDT) in Resistant Hypertension}

Resistant hypertension is defined as blood pressure that remains above therapeutic goal despite the use of three antihypertensive drugs including a diuretics in their maximum therapeutic doses. About one third of patients with arterial hypertension are treatment refractory. The hyperactivity of sympathetic nervous system in the occurrence of treatment resistant long standing hypertension has been established both in animal models and in clinical practice. A new catheter system using radiofrequency energy has been developed, allowing an endovascular approach to renal denervation and providing patients, with resistant hypertension, with a new therapeutic option that is minimally invasive and can be performed rapidly under local anesthesia. With this method the afferent and efferent sympathetic nervous system surrounding the renal artey are ablated precisely keeping the renal artery intact. To date this technique has been evaluated only in open lebel trials highly selected resistant hypertensive patients with suitable renal artery anatomy. RDT will significantly enrich the therapeutic armamentarium for hypertension treatment and control in future. RDT proves to have long lasting beneficial effects, patient would have a choice between interventional therapy and cure of hypertension and life long drug therapy with associated expense and potential side effects. 